\title{
Interactive comment on "Analysis of geomagnetic measurements prior the Maule (2010), Iquique (2014) and Illapel (2015) earthquakes, in the Pacific Ocean sector of the Southern Hemisphere" by Enrique G. Cordaro et al.
}

\section{Patricio Venegas-Aravena}

patricio.venegas@ing.uchile.cl

Received and published: 12 March 2019

\section{Dear referee \# 1,}

Thank you very much for your thoughts and suggestions. We have some typing errors that are easily solvable. It will not happen again. We consider the magnetic field $Z$ and not $\mathrm{Z} / \mathrm{H}, \mathrm{Z} / \mathrm{Z}{ }^{*}$, etc ... since it is crucial to compare with other similar methods. For example 10.1016/j.epsl.2016.12.037 or https://doi.org/10.1016/j.asr.2018.04.043. 
observe the temporal evolution of the magnetic anomalies in periods of time of months or years. So it is much easier to observe if there is an increase in the number of anomalies on dates where earthquakes do not occur. It is important to emphasize that we only consider quiet times so it would not make much sense to show, for example Dst, in these conditions. In the manuscript we show that the spectrogram analysis is not enough, being of low quality in some cases and inconclusive. That is why we decided to focus on the behavior of anomalies. An important point that you comment is about the "globality" of this results. We could use distant magnetometers to corroborate if the behavior of the anomalies is similar to that registered in South America in dates close to large earthquakes. However, we have six years of total registrations in South America. During these six years several earthquakes of magnitude greater than, e.g., Mw7.8 (Nepal 2015 magnitud) occurred around the world. No behavior similar to that obtained for Chilean earthquakes was obtained, so this phenomenon cannot be global in nature. That's why we use the Dobrovolsky area as a reference. Although the deformations are not visible on the surface of the earth from a few hundred kilometers from the future epicenter, it does not necessarily imply that there are no changes of stress under the earth's surface at greater distances. For example, the generation of fractures occurs in the semi-fragile-ductile transition, between 10 and 20 kilometers below the earth's surface. Therefore, small changes in stresses or deformations at this depth may not be detected on the surface. A summary of the electrification in rocks in the non-elastic regime can be seen in 10.1016/j.pce.2003.12.003.

Interactive comment on Ann. Geophys. Discuss., https://doi.org/10.5194/angeo-2019-9, 2019.

Interactive

comment 